\title{
A PROBLEM OF ROBINSON
}

\section{P. R. GARABEDIAN}

We propose to prove in this paper a conjecture of R. M. Robinson which generalizes to domains of connectivity $n \geqq 3$ a result which he proved [3 $]^{1}$ for doubly-connected domains.

Let $D$ be a finite domain of the $z$-plane bounded by $n \geqq 2$ simple, closed, disjoint analytic curves, $C_{1}, \cdots, C_{n}$, whose sum we denote by $C$. Let $z_{0}$ be a point of $D$. We consider the class $\Omega$ of functions $F(z)$ in $D$ with at most one simple pole

$$
F(z)=\frac{\alpha_{F}}{z-z_{0}}+a_{0}+a_{1}\left(z-z_{0}\right)+\cdots,
$$

at $z=z_{0}$, which are regular in the remainder of $D$ and satisfy

$$
\limsup _{z \rightarrow C}|F(z)| \leqq 1
$$

The family $\Omega$ is compact. Thus the real number

$$
\sigma\left(\zeta_{0}\right)=\max _{F \in \Omega}\left|F\left(\zeta_{0}\right)\right|
$$

is defined for every $z_{0} \neq z_{0}$ of $D$.

Since the function $F(z) \equiv 1$ is in the class $\Omega$, it is clear that $\sigma\left(\zeta_{0}\right) \geqq 1$ in $D$. The question raised by Robinson is whether or not there exists in $D$ a nonempty set $A$ on which $\sigma\left(\zeta_{0}\right)=1$. He has solved the problem for the case of an annulus. If the annulus under consideration consists of the points $z$ with $0<r<|z|<1$, and if $r<z_{0}<1$, then he has shown [3] that for $-1<\zeta_{0}<-r$ we have $\sigma\left(\zeta_{0}\right)=1$, while $\sigma\left(\zeta_{0}\right)>1$ in the remainder of $D$. Hence the set $A$ consists here of the segment $-1<\zeta_{0}<-r$. From this result Robinson obtains a highly elegant treatment of questions of the Schwarz lemma type for an annulus.

We shall proceed in the opposite direction. We shall use results obtained in the author's thesis [2] for bounded functions to extend Robinson's theorem to domains of connectivity $n \geqq 3$.

It follows from the methods in the paper cited that there is a unique extremal function $F_{0}\left(z ; \zeta_{0}\right) \in \Omega$ with

$$
F_{0}\left(\zeta_{0} ; \zeta_{0}\right)=\sigma\left(\zeta_{0}\right) \text {. }
$$

If $G(z ; \zeta)$ denotes the Green's function of $D$, we have

Presented to the Society, September 10, 1948; received by the editors May 25, 1948, and, in revised form, August 19, 1948.

${ }^{1}$ Numbers in brackets refer to the bibliography at the end of the paper. 


$$
\log \left|F_{0}\left(z ; \zeta_{0}\right)\right|=G\left(z ; z_{0}\right)-\sum_{\nu=1}^{n-1} G\left(z ; z_{v}\right),
$$

where the points $z_{1}, \cdots, z_{n-1}$ depend on $\zeta_{0}$, and may lie on $C$. When $\sigma\left(\zeta_{0}\right)=1$, one of the $z_{p}$ lies at $z_{0}$ and we have the extremal function

$$
F_{0}\left(z ; \zeta_{0}\right) \equiv 1 \text {. }
$$

The proof of these statements follows so closely the material of Chapter VII of [2] that we omit it here.

The function $F_{0}\left(z ; \zeta_{0}\right)$ depends continuously on the parameter $\zeta_{0}$ in the sense of uniform convergence in every closed subdomain of $D$ not containing $z_{0}$. Also, $\sigma\left(\zeta_{0}\right)$ is a continuous function of $\zeta_{0}$ for $\zeta_{0} \neq z_{0}$ in $D$, and $z_{1}, \cdots, z_{n-1}$ depend continuously on $\zeta_{0}$. For if

$$
\lim _{m \rightarrow \infty} \zeta_{m}=\zeta_{0} \neq z_{0}
$$

then

$$
\begin{aligned}
\sigma\left(\zeta_{0}\right) & =F_{0}\left(\zeta_{0} ; \zeta_{0}\right) \\
& =\lim _{m \rightarrow \infty}\left|F_{0}\left(\zeta_{m} ; \zeta_{0}\right)\right| \\
& \leqq \liminf _{m \rightarrow \infty} F_{0}\left(\zeta_{m} ; \zeta_{m}\right) \\
& =\liminf _{m \rightarrow \infty} \sigma\left(\zeta_{m}\right) .
\end{aligned}
$$

On the other hand, since $\Omega$ is normal and compact, we can pick a subsequence $\zeta_{\mu}^{*}$ of $\zeta_{m}$ and a function $F \in \Omega$ so that

$$
\begin{aligned}
\lim _{\mu \rightarrow \infty} \sigma\left(\zeta_{\mu}^{*}\right) & =\limsup _{m \rightarrow \infty} \sigma\left(\zeta_{m}\right), \\
\lim _{\mu \rightarrow \infty} F_{0}\left(z ; \zeta_{\mu}^{*}\right) & =F(z), \\
\sigma\left(\zeta_{0}\right) & \geqq\left|F\left(\zeta_{0}\right)\right| \\
& =\lim _{\mu \rightarrow \infty}\left|F_{0}\left(\zeta_{0} ; \zeta_{\mu}^{*}\right)\right| \\
& =\lim _{\mu \rightarrow \infty} \lim _{\nu \rightarrow \infty}\left|F_{0}\left(\zeta_{\nu}^{*} ; \zeta_{\mu}^{*}\right)\right| \\
& =\lim _{\mu \rightarrow \infty} F_{\theta}\left(\zeta_{\mu}^{*} ; \zeta_{\mu}^{*}\right)=\limsup _{m \rightarrow \infty} \sigma\left(\zeta_{m}\right) .
\end{aligned}
$$

Therefore $\sigma\left(\zeta_{m}\right) \rightarrow \sigma\left(\zeta_{0}\right)$ as $m \rightarrow \infty$. If $F_{0}\left(z ; \zeta_{m}\right)$ does not approach $F_{0}\left(z ; \zeta_{0}\right)$ as $m \rightarrow \infty$, then there exist subsequences $\zeta_{\mu}^{*}$ and $\zeta_{\mu}^{* *}$ of $\zeta_{m}$ such that

$$
\lim _{\mu \rightarrow \infty} F_{0}\left(z ; \zeta_{\mu}^{*}\right)=F_{1}(z)
$$




$$
\lim _{\mu \rightarrow \infty} F_{0}\left(z ; \zeta_{\mu}^{* *}\right)=F_{2}(z),
$$

where $F_{1}$ and $F_{2}$ are extremal functions in $\Omega$ satisfying

$$
F_{1}\left(\zeta_{0}\right)=F_{2}\left(\zeta_{0}\right)=\sigma\left(\zeta_{0}\right) \text {, }
$$

$F_{1} \not \equiv F_{2}$.

But the extremal function $F_{0}\left(z ; \zeta_{0}\right)$ is unique. Hence we arrive at a contradiction, and it follows that $F_{0}\left(z ; \zeta_{m}\right)$ tends to $F_{0}\left(z ; \zeta_{0}\right)$ as $m$ tends to infinity.

We now choose any curve $\Gamma$ in $D-A$, with its end points both lying at $z_{0}$, which cannot be deformed to a point in $D-A$ in such a way that its end points remain fixed. In topological language, we choose $\Gamma$ to represent an element of the fundamental group of $D-A$ which is not the identity. The assumption that such a curve $\Gamma$ exists will lead us to a contradiction.

Since $\Gamma$ lies entirely inside $D-A$, there exists an $\epsilon>0$ such that $\sigma\left(\zeta_{0}\right) \geqq 1+\epsilon$ for $\zeta_{0} \in \Gamma$. For clearly $\sigma\left(\zeta_{0}\right) \rightarrow \infty$ as $\zeta_{0} \rightarrow z_{0}$, and $\sigma\left(\zeta_{0}\right)$ is continuous and greater than 1 on every closed arc of $\Gamma$ which does not contain $z_{0}$.

Let $\zeta(t)$ be the parametric representation of $\Gamma, 0 \leqq t \leqq 1$. We suppose without loss of generality that $\zeta(t) \neq z_{0}$ for $0<t<1$, while $\zeta(0)$ $=\zeta(1)=z_{0}$. Hence

$$
\bar{\sigma}(t)=\sigma(\zeta(t))
$$

is continuous for $0<t<1$, and $F_{0}(z ; \zeta(t))$ is continuous in its dependence on $t$ for $0<t<1$. We denote by $\Lambda_{t}$ the inverse image of the ray $\bar{\sigma}(t) \leqq w \leqq \infty$ under the mapping $w=F_{0}(z ; \zeta(t))$.

Since $\Gamma$ cannot be shrunk to a point in $D-A$, there is a point $u$ not in $D-A$ such that the order

$$
p=\operatorname{ord}(\Gamma)=\int_{0}^{1} d \arg \{\zeta(t)-u\}
$$

of $\Gamma$ about $u$ is not zero. We denote by $\Gamma\left(t_{0}\right)$ the curve which consists of $\Lambda_{t_{0}}$ plus the arc of $\Gamma$ corresponding to values of $t$ in the interval $t_{0}<t \leqq 1$. Now one end point of $\Lambda_{t_{0}}$ is at $z_{0}$ and the other is at $\zeta\left(t_{0}\right)$. Hence $\Gamma\left(t_{0}\right)$ is a curve whose order $p\left(t_{0}\right)$ about $u$ is well defined.

We maintain that $p\left(t_{0}\right)=p$ for small values of $t_{0}$, say for $0<t_{0}<\delta$. For given any $M>1$, there is a $\delta$ sufficiently small so that if $0<t_{0}<\delta$ we have $\bar{\sigma}\left(t_{0}\right)>M$. Now for any $F \in \Omega$ we have $\log |F(z)| \leqq G\left(z ; z_{0}\right)$. Hence if $0<t_{0}<\delta$, then $\Lambda_{t_{0}}$ lies in a neighborhood $G\left(z ; z_{0}\right)>\log M$ of $z_{0}$, and such a neighborhood becomes arbitrarily small for sufficiently large $M$. Therefore it follows that $p\left(t_{0}\right)=p$ for $0<t_{0}<\delta$.

Similarly, we find there is a $\Delta>0$ such that $p\left(t_{0}\right)=0$ for $1-\Delta<t_{0}<1$. 
Now $p \neq 0$. Therefore $p\left(t_{0}\right)$ cannot be continuous in the interval

$$
\delta / 2 \leqq t_{0} \leqq 1-\Delta / 2,
$$

since $p\left(t_{0}\right)$ is an integral-valued function.

The residue of $F_{0}(z ; \zeta(t))$ at $z=z_{0}$ is bounded away from zero in the interval (17), since $\bar{\sigma}(t) \geqq 1+\epsilon$ there and $F_{0}(z ; \zeta(t))$ depends continuously on $t$. Also, $F_{0}(z ; \zeta(t))$ assumes each value $w$ with $|w|>1$ precisely once in $D$, and hence its inverse function is uniquely defined for $|w|>1$. Indeed, the inverse function,

$$
z-z_{0}=H(w ; \zeta(t))=\frac{\beta_{t}}{w}+\frac{b_{2}}{w^{2}}+\frac{b_{3}}{w^{3}}+\cdots, \quad|w|>1,
$$

is schlicht for $|w|>1$ and its distortion $\beta_{t}$ at infinity is bounded away from zero and infinity, since $\beta_{t}=\alpha_{F_{0}}, F_{0}=F_{0}(z ; \zeta(t))$.

It follows that $\Lambda_{t}$ depends continuously on $t$ in the sense of uniform convergence of its parametric representation. Therefore $p\left(t_{0}\right)$ is continuous in the interval (17), and we arrive at a contradiction.

We have proved the following theorem.

Theorem. Let $\Omega$ be the class of functions $F(z)$ in D which satisfy (2) and have at most one simple pole (1) at $z_{0} \in D$, and let $\sigma\left(\zeta_{0}\right)$ be defined by (3). If $A \subset D$ is the set of points $\zeta_{0} \in D$ such that $\sigma\left(\zeta_{0}\right)=1$, then the region $D-A$ is simply-connected.

It is of interest to consider, following Robinson, the question of how many poles we must allow the functions in our class to have in order that the set $A$ be vacuous. To be precise, we consider the class $\Omega\left(z_{1}, \cdots, z_{m}\right)$ of functions $F(z)$ satisfying (2) and regular in $D$ except for possible simple poles at $z_{1}, \cdots, z_{m}$. If several of the $z_{j}$ coincide, we allow the poles in question to have correspondingly increased multiplicities. We define the function $\sigma\left(\zeta_{0}\right)$ by (3) with the new class $\Omega=\Omega\left(z_{1}, \cdots, z_{m}\right)$, and we denote by $A\left(z_{1}, \cdots, z_{m}\right)$ the set of points $\zeta_{0} \in D$ with $\sigma\left(\zeta_{0}\right)=1$.

If $m \geqq n$, the set $A\left(z_{1}, \cdots, z_{m}\right)$ is seen to be vacuous by the results of the papers $[1,2]$. Indeed, the variation of zeros introduced there shows that the extremal function $F_{0} \in \Omega\left(z_{1}, \cdots, z_{m}\right)$ with $F_{0}\left(\zeta_{0}\right)$ $=\sigma\left(\zeta_{0}\right)$ satisfies an identity

$$
\log \left|F_{0}(z)\right|=\sum_{j=1}^{m} G\left(z ; z_{j}\right)-\sum_{j=1}^{n-1} G\left(z ; \zeta_{j}\right),
$$

where the points $\zeta_{1}, \cdots, \zeta_{n-1}$ depend on $\zeta_{0}$. Since the number of zeros $\zeta_{j}$ of the extremal function is less than the number of poles $z_{j}$, 
the extremal function is not constant, and we have $\sigma\left(\zeta_{0}\right)>1$.

The same type of argument can be used to show that, in general, if $m=n-1 \geqq 2$, then $A\left(z_{1}, \cdots, z_{m}\right)$ is vacuous. However, in every domain of connectivity $n \geqq 2$ there exist points $z_{1}, \cdots, z_{n-1}$ such that the set $A\left(z_{1}, \cdots, z_{n-1}\right)$ for the class $\Omega\left(z_{1}, \cdots, z_{n-1}\right)$ is not empty. For let $z_{1}^{*}, \cdots, z_{n-1}^{*}$ be the critical points of the Green's function, $G\left(z ; \zeta_{0}\right)$, of $D$ with normalization point at $\zeta_{0} \in D$. We maintain that $\zeta_{0}$ is in the set $A\left(z_{1}^{*}, \cdots, z_{n-1}^{*}\right)$.

Indeed, let $F \in \Omega\left(z_{1}^{*}, \cdots, z_{n-1}^{*}\right)$, and set

$$
p^{\prime}(z)=\frac{\partial G\left(z ; \zeta_{0}\right)}{\partial x}-i \frac{\partial G\left(z ; \zeta_{0}\right)}{\partial y}, \quad z=x+i y_{0}
$$

Then $^{2}$

$$
\left|F\left(\zeta_{0}\right)\right|=\left|-\frac{1}{2 \pi i} \oint_{C} F(z) p^{\prime}(z) d z\right| \leqq \frac{1}{2 \pi} \oint_{C}|d p(z)|=1 .
$$

The technique of contour integration which we use here is precisely the same as that developed in the paper [2].

We introduce the harmonic functions $\omega_{i}(z)$ known as the harmonic measures of the $C_{i}$ in $D, i=1, \cdots, n$. The function $\omega_{i}(z)$ is harmonic in $D$ and assumes the boundary values 1 on $C_{i}$ and the boundary values 0 on the remainder of $C$. We see that any function

$$
h(z)=G\left(z ; \zeta_{0}\right)+\sum_{i=1}^{n-1} \lambda_{i} \omega_{i}(z)
$$

can be used in the role of $G\left(z ; \zeta_{0}\right)$ in the argument of the preceding paragraph, provided the normal derivative of $h(z)$ is non-negative on $C$.

We now return to the consideration of $\Omega=\Omega\left(z_{0}\right)$. Suppose that $z_{0}$ is a critical point of $h(z)$. Then we can apply the method embodied in the relation (21) to functions $F \in \Omega$ in order to show that $\zeta_{0} \in A$. If $n \geqq 3$, it will in general be possible to choose the parameters $\lambda_{1}^{*}, \cdots, \lambda_{n-1}^{*}$ in such a way that

$$
h^{*}(z)=G\left(z ; \zeta_{0}^{*}\right)+\sum_{i=1}^{n-1} \lambda_{i}^{*} \omega_{i}(z)
$$

has a critical point at $z_{0}$ and has a non-negative normal derivative on

\footnotetext{
${ }^{2}$ Here and in (25) we apply Cauchy's theorem to contour integrals over $C$ of the function $F$. This is permissible, since $F$ has limits in angle almost everywhere on $C$. These limits can be used in the contour integrals (21) and (25), as is seen from the Lebesgue convergence theorem.
} 
$C$, for all values of $\zeta_{0}^{*}$ in some small neighborhood of $\zeta_{0}$. Indeed, this is possible if all $n-1$ of the critical points of (22) lie in $D$ and the vectors

$$
\left.\left[\frac{\partial \omega_{i}(z)}{\partial x}-i \frac{\partial \omega_{i}(z)}{\partial y}\right]\right|_{z=z_{0}}, \quad i=1, \cdots, n-1,
$$

do not lie on a line through the origin. Thus we shall have

$$
\begin{aligned}
\left|F\left(\zeta_{0}^{*}\right)\right| & =\left|-\frac{1}{2 \pi i} \oint_{C}\left[\frac{\partial h^{*}}{\partial x}-i \frac{\partial h^{*}}{\partial y}\right] F(z) d z\right| \\
& \leqq \frac{1}{2 \pi} \oint_{C} \frac{\partial h^{*}}{\partial \nu} d s=1,
\end{aligned}
$$

where $\nu$ represents the inner normal to $C$.

Therefore we can conclude that if $n \geqq 3$ the set $A=A\left(z_{0}\right)$ has, in general, interior points. There will be special cases in which this is not so, because our conditions upon the parameters $\lambda_{1}^{*}, \cdots, \lambda_{n-1}^{*}$ in order that $h^{*}(z)$ have a critical point at $z_{0}$ and a non-negative normal derivative on $C$ can degenerate. This happens, for example, when $D$ is an annulus $r<|z|<1$ with slits along the segment $-1<z$ $<-r$, and $z_{0}>0$. For this case of symmetry one sees from Robinson's original theorem for circular rings [3] that the set $A$ lies on the real axis.

It is clear that further progress in the investigation of the present set of problems can be achieved by finding precise information concerning the relative locations of the singularities and critical points of the harmonic functions (23) with non-negative normal derivative on $C$.

\section{BIBLIOGRAPHY}

1. L. Ahlfors, Bounded analytic functions, Duke Math. J. vol. 14 (1947) pp. 1-11.

2. P. R. Garabedian, Schwarz's lemma and the Szegö kernel function, Thesis, Harvard University, 1948.

3. R. M. Robinson, Analytic functions in circular rings, Duke Math. J. vol. 10 (1943) pp. 341-354.

\section{HARVARD UNIVERSITY}

